\title{
Home tracheotomy mechanical ventilation in patients with amyotrophic lateral sclerosis: causes, complications and 1-year survival
}

\author{
Jesús Sancho, ${ }^{1,2}$ Emilio Servera, ${ }^{1,2,3}$ José Luis Díaz, ${ }^{1,2,3}$ Pilar Bañuls, ${ }^{1,2}$ Julio Marín ${ }^{2,3}$
}

\section{See Editorial, p 932}

- Additional materials are published online only. To view these files please visit the journal online (http://thorax.bmj. com)

${ }^{1}$ Respiratory Care Unit, Respiratory Medicine Department, Hospital Clínico Universitario, Valencia, Spain ${ }^{2}$ Research Group for Respiratory Problems in Neuromuscular Diseases, Fundación para la Investigación HCUV-INCLIVA, Valencia, Spain

${ }^{3}$ Universitat de Valencia, Valencia, Spain

\section{Correspondence to}

Dr Jesús Sancho, Respiratory Care Unit, Respiratory Medicine Department, Hospital Clínico Universitario, Avd Blasco Ibañez 17.46010 Valencia, Spain; cchinesta@eresmas.com

Received 31 January 2011 Accepted 26 May 2011 Published Online First 21 June 2011

\begin{abstract}
Background Home tracheotomy mechanical ventilation (HTMV) can prolong survival in patients with amyotrophic lateral sclerosis (ALS) when non-invasive ventilation (NIV) fails, but knowledge about HTMV is scarce. The aim of this study was to determine the causes of tracheotomy and the main issues of 1-year HTMV in a cohort of patients with ALS.
\end{abstract}

Methods A prospective study of all patients needing HTMV was performed in a referral respiratory care unit (RCU) from April 2001 to January 2010. Patients' informed decisions about HTMV were fully respected. Caregivers were trained and could telephone the RCU. Hospital staff made home visits.

Results All patients $(n=116)$ agreed to participate and a tracheotomy was needed for 76 , mainly due to bulbar dysfunction. Of the 38 who had a tracheotomy, in 21 it was performed in an acute setting and in 17 as a nonemergency procedure. In 19 patients the tracheotomy was related to the inadequacy of mechanically assisted coughing (MAC) to maintain normal oxygen saturation. During HTMV, 19 patients required hospitalisation, 12 with respiratory problems. The 1-year survival rate was $78.9 \%$, with a mean survival of 10.39 months $195 \% \mathrm{Cl}$ 9.36 to 11.43). Sudden death was the main cause of death $(n=9)$ and only one patient died from respiratory causes. No predictive factors for survival were found.

Conclusions Besides NIV inadequacy, the ineffectiveness of mechanically assisted coughing appears to be a relevant cause of tracheotomy for patients with ALS with severe bulbar dysfunction. Patients choosing HTMV provided by a referral RCU could have a good 1-year survival rate, respiratory problems being the main cause of hospitalisation but not of death.

Although respiratory problems related to inspiratory and expiratory muscle weakness and to upper airway muscle dysfunction are the main cause of morbidity and mortality in patients with amyotrophic lateral sclerosis (ALS), ${ }^{1}$ the use of appropriate respiratory muscle aids can change the course of the disease dramatically. Non-invasive ventilation (NIV) and mechanically assisted coughing (MAC) can prolong survival, ${ }^{2}{ }^{3}$ reduce hospitalisations, ${ }^{2}$ improve quality of life $^{3}$ and relieve symptoms in patients with ALS. ${ }^{2}{ }^{3}$ Nevertheless, when bulbar dysfunction (BD) reaches a critical level, non-invasive muscle aids become inadequate ${ }^{2} 4$ and it is essential to perform a tracheotomy or to intensify appropriate palliative care.

Home tracheotomy mechanical ventilation (HTMV) can prolong survival for patients with advanced ALS, ${ }^{5}$ and this is the procedure of choice for those who want to continue living. ${ }^{67}$ However, when one examines the guidelines, the paucity of information on HTMV (the best time to start, the right procedures, main complications, real burdens, etc) is clearly inferior to the information available for other ALS management procedures. ${ }^{6} 7$ This lack of information could be related to the small quantity and the protocol limitations of the studies available on HTMV in ALS. Although they provide new and valuable information, the existing studies are retrospective, ${ }^{8}$ the HTMV management procedures are not well described ${ }^{5-10}$ and most of the patients included have had an unplanned tracheotomy. $^{8}{ }^{10-12}$ Despite the fact that the expert guidelines ${ }^{6713}$ have for some years highlighted the need to ensure that patients are fully informed before making a decision about HTMV, the available data ${ }^{8-12}$ show that, in clinical practice, this rarely occurs.

The weakness of the available knowledge about HTMV has a negative impact on physicians' decision-making and on the quality of the information with which patients are provided, and this could be one of the reasons for some treating physicians lessening the patients' autonomy about HTMV decisions. ${ }^{12} 14$ In the current scenario the best clinical practice for this therapeutic approach-one that will have a dramatic effect on the patient's future and that of their relatives-is difficult to achieve. In order to contribute to scientific knowledge on this critical issue in the management of patients with ALS, the aims of this study were to determine the causes for tracheotomy, to analyse the main post-tracheotomy complications, to determine the causes of death and to search for the predictive factors for 1-year survival in a cohort of patients with ALS with HTMV under the care of a referral respiratory care unit (RCU) and on the basis of informed shared decision-making.

\section{MATERIAL AND METHODS}

This prospective study was performed in a referral ALS unit from April 2001 to January 2010 and included, after informed consent, all the patients diagnosed with probable or definite ALS ${ }^{15}$ managed at our RCU. Exclusion criteria were refusal to participate, pulmonary disease or another disease with a poor prognosis. The study protocol was approved by the hospital's ethics committee.

A pulmonologist informed all the patients and relatives about the respiratory problems of ALS and the circumstances in which a tracheotomy might be necessary, its effects on life support and on the progression of the disease and the associated 
burdens. Treatment decisions were taken on the basis of informed shared decision-making ${ }^{16}$ and advanced directives were recorded.

Clinical and functional assessments were made in the 3-month period prior to the tracheotomy as described previously. ${ }^{16-19}$ Patients were also evaluated with the revised amyotrophic lateral sclerosis functional rating scale (ALSRFS-R) ${ }^{17}$ and the Norris scale bulbar subscore (NBS). ${ }^{18}$ When necessary, non-invasive muscle aids were applied. ${ }^{6} 13$

\section{A tracheotomy was considered necessary for the following:}

1. To ensure adequate alveolar ventilation when patients were unable to subjectively tolerate NIV provided by volume ventilators and delivered via nasal, oronasal and mouth interfaces or when NIV could not provide adequate alveolar ventilation. This could happen:

a. in 'non-acute conditions' when the inadequacy of NIV makes a tracheotomy a mandatory but not an immediately life-saving procedure, with such failure becoming evident by the presence of one or more of the following: dyspnoea, disrupted sleep, diurnal somnolence, headache, altered cognitive status, oxygen saturation $\left(\mathrm{SpO}_{2}\right)<95 \%$, arterial carbon dioxide tension $>50 \mathrm{~mm} \mathrm{Hg}^{20}$;

b. in 'acute settings' after endotracheal intubation (ETI) needed to maintain life during a life-threatening situation related to an acute respiratory tract infection (ARTI). Here, NIV could not ameliorate hypercapnia, nor could it relieve respiratory distress, lethargy or rapidly decreasing $\mathrm{Spo}_{2}$.

2. To manage respiratory secretions. A tracheotomy was also needed when MAC (Cough-Assist, Philips-Respironics International, Murrysville, Pennsylvania, USA) was inadequate to remove airway secretions, causing $\mathrm{SpO}_{2}<95 \%$, and/or patients felt continuously encumbered or dyspnoeic.

Tracheotomies were performed percutaneously in the ICU and patients returned to our RCU within $24 \mathrm{~h}$. Once the patients were medically stable and caregivers had been appropriately trained, they were transferred home. ${ }^{21}$

HTMV was delivered with a volume cycled ventilator through an uncuffed tube but, if air leaks caused discomfort or made mechanical ventilation ineffective, cuffed tubes were used. Fenestrated tubes were employed for patients with ventilator-free time. Respiratory secretions were managed with MAC through a tracheotomy tube as previously described. ${ }^{22}$

Caregivers could contact the RCU by telephone. A nurse from the RCU visited the patients at home once every 2 weeks and a pulmonologist every 3 months.

Additional details of the methods and equipment are provided in the online supplement.

\section{Statistical analysis}

Binary and categorical variables were summarised using frequency counts and percentages. Continuous normally distributed variables were expressed as mean \pm SD and those not normal as median (IOR). Data comparisons were performed using the Student $t$ test and the Mann-Whitney test for normally and not normally distributed data, respectively. Dichotomic variables were compared with the $\chi^{2}$ test. Longterm survival was assessed with Kaplan-Meier charts, applying the log-rank test for between-group comparisons. Cox univariate and multivariate stepwise proportional hazards were used to study the prognostic factors for survival. The multivariate analysis model, adjusted for sex, age and site of ALS onset, included those variables that exhibited a significant association in the univariate model. Statistical significance was taken as $\mathrm{p}<0.05$.

\section{RESULTS}

From April 2001 to January 2010, 116 patients with ALS were managed in our RCU. All of them agreed to participate in the study.

During this period tracheotomies were needed for 76 patients. The demographic and clinical characteristics of these patients in the 3-month period prior to the need for tracheotomy are shown in table 1 . Of these patients, $38(50 \%)$ agreed to the procedure and 38 refused it and went on to receive appropriate palliative care. The characteristics of the patients who agreed to the tracheotomy did not differ from those who rejected it (table 1). No patient died as a result of the tracheotomy procedure or related problems.

In 21 patients (11 of whom were already using NIV at home) the tracheotomy was performed after an urgent ETI carried out during an ARTI. Apart from one patient who refused NIV and

Table 1 Demographic data and clinical characteristics for the whole group of patients with ALS, for those who refused a tracheotomy and for those who agreed to it

\begin{tabular}{|c|c|c|c|c|}
\hline & All patients & Refused tracheotomy & Agreed to tracheotomy & p Value \\
\hline $\operatorname{Sex}(M / F)$ & $30 / 46$ & $14 / 24$ & $16 / 22$ & 0.639 \\
\hline Age (years) & $66.00 \pm 9.18$ & $66.97 \pm 9.22$ & $65.03 \pm 9.15$ & 0.359 \\
\hline BMI $\left(\mathrm{kg} / \mathrm{m}^{2}\right)$ & $25.48 \pm 3.67$ & $25.46 \pm 3.91$ & $25.51 \pm 3.46$ & 0.057 \\
\hline Spinal/bulbar onset & $42 / 34$ & $23 / 15$ & $19 / 19$ & 0.356 \\
\hline Time from ALS onset to tracheotomy (months) & $37.97 \pm 32.11$ & $35.26 \pm 20.58$ & $41.29 \pm 42.36$ & 0.442 \\
\hline ALSFRS-R & $11.63 \pm 3.85$ & $11.02 \pm 4.05$ & $12.21 \pm 3.11$ & 0.910 \\
\hline NBS & $11.10 \pm 8.70$ & $11.24 \pm 8.62$ & $10.38 \pm 9.01$ & 0.693 \\
\hline FVC (I) & $1.01 \pm 0.51$ & $1.03 \pm 0.50$ & $0.99 \pm 0.52$ & 0.745 \\
\hline$\%$ FVC $(\%)$ & $39.53 \pm 18.46$ & $39.28 \pm 17.93$ & $39.75 \pm 19.20$ & 0.924 \\
\hline MIC (I) & $1.28 \pm 0.63$ & $1.31 \pm 0.61$ & $1.26 \pm 0.66$ & 0.743 \\
\hline PCF (I/s) & $2.24 \pm 1.06$ & $2.37 \pm 1.06$ & $2.13 \pm 1.07$ & 0.381 \\
\hline $\mathrm{PCF}_{\mathrm{MIC}}(\mathrm{l} / \mathrm{s})$ & $2.55 \pm 1.17$ & $2.71 \pm 1.13$ & $2.40 \pm 1.20$ & 0.320 \\
\hline $\mathrm{PCF}_{\mathrm{MI}-\mathrm{E}}(\mathrm{l} / \mathrm{s})$ & $3.13 \pm 0.89$ & $3.15 \pm 0.64$ & $3.12 \pm 1.07$ & 0.890 \\
\hline PImax $\left(\mathrm{cm} \mathrm{H} \mathrm{H}_{2} \mathrm{O}\right)$ & $-32.09 \pm 17.46$ & $-36.08 \pm 18.47$ & $-28.53 \pm 15.99$ & 0.117 \\
\hline PEmax $\left(\mathrm{cm} \mathrm{H}_{2} \mathrm{O}\right)$ & $44.33 \pm 23.09$ & $44.53 \pm 22.81$ & $44.14 \pm 25.29$ & 0.952 \\
\hline
\end{tabular}

Data are expressed as means $\pm S D$ for continuous variables and number for categorical variables.

ALS, amyotrophic lateral sclerosis; ALSFRS-R, revised amyotrophic lateral sclerosis functional rating scale; BMI, body mass index; MIC, maximum insufflation capacity; FVC, forced vital

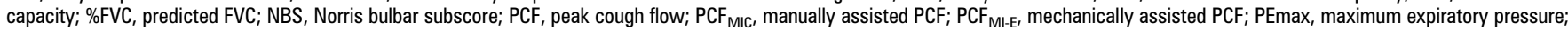
Plmax, maximum inspiratory pressure. 
chose a tracheotomy as their first option, the tracheotomy was performed after the failure of managing the situation noninvasively: inadequacy of use of NIV to maintain normal alveolar ventilation ( $n=8,38.1 \%)$; ineffectiveness of MAC to remove airway secretions $(n=3,14.3 \%)$; or a combination of the two $(\mathrm{n}=9,42.9 \%)$.

In 17 patients the tracheotomy was carried out as a nonemergency procedure. Prior to the tracheotomy, nine $(52.9 \%)$ were using non-invasive respiratory muscle aids at home (one was managed with NIV, four with MAC, and four with both), while eight patients $(47.1 \%)$ did not receive these treatments. The causes leading to tracheotomy in patients previously using non-invasive muscle aids were: inadequacy of NIV to maintain satisfactory alveolar ventilation $(n=1)$; rapid decrease in adequacy of MAC $(n=1)$; and a combination of the two $(n=1)$. In the eight patients not using non-invasive respiratory muscle aids at home, the immediate causes for performing a tracheotomy were: refusal of NIV and agreement to tracheotomy ventilation when assisted ventilation was needed $(n=1)$; inability to achieve effective NIV, probably due to severe BD $(n=2)$; and inefficacy of MAC $(n=4)$. Finally, for one patient, following their stated wishes, the tracheotomy was brought forward and carried out for the purposes of general anaesthesia required for scheduled surgical procedures not related to ALS.

The demographic and clinical characteristics assessed prior to the need for a tracheotomy are shown in table 2. No statistical differences were found between the patients who underwent a non-urgent tracheotomy and those who underwent this procedure during an acute episode. One patient for whom a tracheotomy was indicated due to ineffective NIV because of excessive non-compensable air leaks did not have BD (NBS 39), but most of the patients had severe BD (NBS 11.24 \pm 8.62 ).

\section{Home discharge and follow-up}

The mean \pm SD post-tracheotomy RCU stay was $26.8 \pm 9.9$ days. Seventeen patients (44.7\%) were discharged with full-time HTMV and 21 with part-time HTMV (17.89 $\pm 6.55 \mathrm{~h} /$ day). Statistical differences between patients with full-time ventilation and those with part-time ventilation at discharge were found only in ALSRFS-R (9.35 \pm 3.79 vs $12.38 \pm 3.81$, respectively, $\mathrm{p}=0.02$ ).

Except for the abovementioned patient for whom NIV failed because of leaks without obvious BD (NBS 39), the rest of the patients were sent home with nutrition by percutaneous endoscopic gastrostomy.

Nineteen patients $(50 \%)$ required hospitalisation during the first year, with a total of 31 hospital admissions; the average number of hospitalisations per patient was $0.82 \pm 0.98$ (range $0-3)$. The main cause of hospitalisation (58\%) was ARTI episodes requiring intravenous antibiotic therapy against multidrug-resistant bacteria or the need for oxygen therapy due to a fall in $\mathrm{SpO}_{2}$. Pneumonia was the cause of $35 \%$ of the ARTIs. Apart from ARTIs needing hospitalisation, the patients had a mean of $1.34 \pm 0.45$ ARTIs managed successfully at home. The most frequent organism colonising the airway was Pseudomonas aeruginosa (found in $57 \%$ of patients).

The second most common cause of hospitalisation $(n=6)$ was for psychiatric assessment or treatment, but all these patients were hospitalised to avoid the inconvenience of travelling for them and their carers rather than because of the seriousness of their emotional problems. The diagnoses were: dyssomnia $(n=1)$; adjustment disorder with depressed mood $(n=3)$; and adjustment disorder with anxiety $(n=2)$. The patients were treated with psychotherapy and drugs, with the patient with dyssomnia being hospitalised once and the others twice for the adjustment of treatment.

The third most common cause was tracheotomy management problems, with one patient hospitalised to manage a stomal haemorrhage and another to treat a granuloma from the tracheal wall occluding the distal tip of the tube.

Other causes of hospitalisation (affecting one patient in each case) were: the need for an exhaustive medical examination, a head injury, acute gastroenteritis, acute cholecystitis and severe hypokalaemia.

\section{Causes of death and 1-year survival analysis}

Eight patients died during the year, with the most frequent cause being sudden death at home $(n=4)$. Other causes of death

Table 2 Demographic data and clinical characteristics for patients with ALS with a tracheotomy performed during an acute episode and those with a non-urgent tracheotomy

\begin{tabular}{|c|c|c|c|}
\hline & Acute $(n=21)$ & Non-acute $(n=17)$ & p Value \\
\hline $\operatorname{Sex}(M / F)$ & $8 / 13$ & $8 / 9$ & 0.578 \\
\hline Age (years) & $63.52 \pm 10.55$ & $66.88 \pm 6.92$ & 0.267 \\
\hline $\mathrm{BMI}\left(\mathrm{kg} / \mathrm{m}^{2}\right)$ & $27.17 \pm 3.28$ & $23.76 \pm 2.77$ & 0.052 \\
\hline Time from ALS onset to tracheotomy (months) & $42.26 \pm 47.95$ & $39.75 \pm 26.02$ & 0.875 \\
\hline ALSFRS-R & $10.95 \pm 3.91$ & $11.11 \pm 4.32$ & 0.902 \\
\hline NBS & $11.76 \pm 8.41$ & $10.59 \pm 9.09$ & 0.682 \\
\hline MIC (I) & $1.16 \pm 0.72$ & $1.37 \pm 0.58$ & 0.373 \\
\hline PCF $(1 / \mathrm{s})$ & $1.89 \pm 1.00$ & $2.40 \pm 1.12$ & 0.187 \\
\hline $\mathrm{PCF}_{\mathrm{MIC}}(\mathrm{l} / \mathrm{s})$ & $2.15 \pm 1.22$ & $2.70 \pm 1.15$ & 0.206 \\
\hline $\mathrm{PCF}_{\mathrm{MI}-\mathrm{E}}(\mathrm{l} / \mathrm{s})$ & $2.77 \pm 0.87$ & $3.51 \pm 1.17$ & 0.058 \\
\hline PImax $\left(\mathrm{cm} \mathrm{H}_{2} \mathrm{O}\right)$ & $-29.28 \pm 16.44$ & $-27.78 \pm 16.11$ & 0.809 \\
\hline
\end{tabular}

Data are expressed as means $\pm S D$ for continuous variables and number for categorical variables.

ALS, amyotrophic lateral sclerosis; ALSFRS-R, revised amyotrophic lateral sclerosis functional rating scale; BMl, body mass index; FVC, forced vital capacity; \%FVC, predicted FVC; MAC,

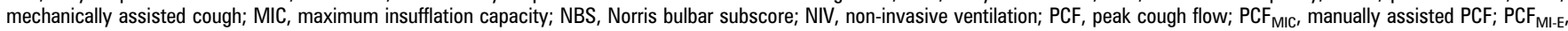
mechanically assisted PCF; PEmax, maximum expiratory pressure; PImax, maximum inspiratory pressure. 
were: ventilation withdrawal $(n=1)$; acute abdomen attended to at home by the emergency medical service, with the patient dead on arrival at hospital $(\mathrm{n}=1)$; cardiac arrest during an acute heart failure attended to at home by the GP with the patient dead on arrival at hospital $(n=1)$; and respiratory tract infection, having refused to be taken to hospital $(n=1)$.

These figures give a survival rate of $78.9 \%$, with a mean survival of 10.39 months ( $95 \%$ CI 9.36 to 11.43 ). No statistical differences were found in survival between patients with ALS in whom a tracheotomy was performed during an acute episode (survival rate $85.7 \%$, survival time 10.76 months, 95\% CI 9.46 to 12.06 ) and those in a non-acute episode (survival rate $70.0 \%$, survival time 9.94 months, $95 \%$ CI 8.31 to 11.58 ). The mean survival for patients refusing a tracheotomy was 0.83 months (95\% CI 0.60 to 1.06$)$. Statistical differences were found for survival between patients refusing a tracheotomy and those agreeing to it $(\mathrm{p}<0.0001)$.

In the univariate and multivariate analysis, no variable was related to the rate of survival (table 3 )

\section{DISCUSSION}

Our findings show that most patients with ALS from a referral unit for whom the informed shared decision was to receive HTMV after NIV failure can be successfully managed at home during the following year. Moreover, we found that although the main cause for hospital admissions in these patients was ARTIs, the main cause of death was not related to respiratory problems.

In ALS, severe BD allows respiratory problems to appear, ${ }^{2} 4$ and it was present in most of our patients at the moment when non-invasive aids failed and a tracheotomy was essential. For $50 \%$ of patients this failure was related-either as the direct or associated cause along with the inability of NIV to provide adequate alveolar ventilation-to our inability to manage respiratory secretions despite the use of MAC, whose effectiveness was clearly affected by BD both in acute and non-acute settings. Most experts highlight the importance of managing

Table 3 Univariate analysis of 1-year survival after tracheotomy in patients with ALS

\begin{tabular}{|c|c|c|}
\hline & HR $(95 \%$ Cl) & p Value \\
\hline Sex & $0.725(0.17$ to 3.03$)$ & 0.421 \\
\hline Age (65.02 years) & $1.042(0.94$ to 1.14$)$ & 0.389 \\
\hline BMI $\left(25.51 \mathrm{~kg} / \mathrm{m}^{2}\right)$ & $1.188(0.94$ to 1.49$)$ & 0.137 \\
\hline Onset & $0.279(0.05$ to 1.38$)$ & 0.119 \\
\hline ALSFRS-R (11.02) & $0.906(0.75$ to 1.08$)$ & 0.287 \\
\hline NBS (11.23) & $0.957(0.86$ to 1.06$)$ & 0.418 \\
\hline Time from ALS onset (41.29 months) & $0.997(0.97$ to 1.01$)$ & 0.737 \\
\hline FVC (0.99 I) & 0.701 (0.15 to 3.27$)$ & 0.651 \\
\hline$\% F V C(39.75 \%)$ & $0.988(0.95$ to 1.02$)$ & 0.552 \\
\hline MIC (1.26 I) & $1.264(0.43$ to 3.64$)$ & 0.665 \\
\hline $\operatorname{PCF}(2.13 \mathrm{l} / \mathrm{s})$ & $0.923(0.45$ to 1.85$)$ & 0.821 \\
\hline $\mathrm{PCF}_{\mathrm{MIC}}(2.40 \mathrm{l} / \mathrm{s})$ & $0.886(0.47$ to 1.65$)$ & 0.704 \\
\hline $\mathrm{PCF}_{\mathrm{MI-E}}(3.12 \mathrm{l} / \mathrm{s})$ & $1.068(0.55$ to 2.05$)$ & 0.845 \\
\hline PImax $\left(-28.53 \mathrm{~cm} \mathrm{H}_{2} 0\right)$ & $1.011(0.96$ to 1.06$)$ & 0.686 \\
\hline PEmax $\left(44.14 \mathrm{~cm} \mathrm{H}_{2} \mathrm{O}\right)$ & 0.991 (0.95 to 1.02$)$ & 0.566 \\
\hline Hours with MV (17.89 h) & 1.164 (0.98 to 1.38$)$ & 0.080 \\
\hline Home NIV prior to tracheotomy & $1.000(0.48$ to 2.07$)$ & 1.000 \\
\hline Home MAC prior to tracheotomy & $1.000(0.48$ to 2.05$)$ & 1.000 \\
\hline
\end{tabular}

ALS, amyotrophic lateral sclerosis; ALSFRS-R, revised amyotrophic lateral sclerosis functional rating scale; BMI, body mass index; FVC, forced vital capacity; \%FVC, predicted FVC; MAC, mechanically assisted cough; MIC, maximum insufflation capacity; NBS, Norris bulbar subscore; NIV, non-invasive ventilation; $\mathrm{PCF}$, peak cough flow; $\mathrm{PCF}_{\mathrm{MIC}}$, manually assisted PCF; PCF $_{\mathrm{MI}-\mathrm{E}}$, mechanically assisted PCF; PEmax, maximum expiratory pressure; PImax, maximum inspiratory pressure. secretions in ALS, ${ }^{6}$ but what has not been described in previous studies is the real incidence of airway secretions as the cause of non-invasive management failure and of the need for ETI and a subsequent tracheotomy. ${ }^{2311}$ Our results confirm our previous findings ${ }^{4}$ and show that, unlike what happens at earlier stages of the disease, for patients with serious BD according to the NBS score, MAC techniques do not achieve effective peak cough flow values. The accumulation of respiratory secretions may therefore make a tracheotomy necessary to remove them. This must be taken into account to ensure the tracheotomy is not inappropriately delayed.

With regard to the ratio of patients requiring a tracheotomy in acute versus non-acute settings, our results (55.26\% vs $44.74 \%$ ) are similar to those of Chio et $a l^{9}(44.02 \%$ vs $55.97 \%)$ and differ greatly from those of Cazzolli and Oppenheimer ${ }^{12}$ (92\% vs $8 \%$ ).

Nowadays, starting HTMV should no longer necessarily be considered as the 'beginning of the end' for these patients. When the appropriate medical resources are in place and the patients wish to continue living, the old mentality of focusing only on palliative care-which is still common in the management of this disease-must make way for 'high technology and compassionate care' (Dr Anthony Oppenheimer, personal communication, 2003). Our 1-year survival rate of $79 \%$ is higher than that for glioblastoma multiforme treatment $(62 \%)^{23}$ and close to that of lung transplants (82.7\%), ${ }^{24}$ two high technology therapeutic procedures which are consolidated within standard medical practice. Of course, HTMV and palliative care must not be considered as binary mutually exclusive alternatives. While providing high technology care to maintain life, providing the best compassionate care to mitigate symptoms and to relieve suffering is essential. ${ }^{7}$

The need to receive psychiatric assessment and treatment was the second most common cause of admission to hospital in our study but, if there had been a psychiatric home care service, such hospitalisations would not have been necessary. Studies show a wide range in the incidence of depression in patients with ALS with $\mathrm{HTMV}^{8}{ }^{10}$ but there are no data regarding the importance of depression as a cause of hospitalisation. Our previous data ${ }^{25}$ agree with those of Vianello et al, ${ }^{8}$ showing that patients with a tracheotomy do not have higher levels of depression than those with NIV and those who do not require mechanical ventilation. In fact, the severity of depressive symptoms in patients with ALS is not related to the loss of function that comes with the progression of the disease, but rather to social support, coping capacity and independence. ${ }^{26} 27$ This underlines the importance of honest communication with patients and relatives in order to make clear the future scenario with $\mathrm{HTMV}^{28}$ and to continue clarifying any further uncertainty. ${ }^{7}$

One limitation of this study is that we did not include an assessment of patients' and caregivers' quality of life or satisfaction with life in the protocol of this study. We are collecting this important information in our current research.

With a rate of around $45 \%$ of reported deaths, ARTI is by far the most common cause of mortality in patients with ALS with HTMV in previous studies. 58911 However, our results show dramatic differences because, while ARTI was the primary cause of hospitalisation, only one patient died as a result of an ARTI, having refused to be taken to hospital. Although we have no definitive explanation for these differences, in our protocol the prevention and prompt management of ARTI was a target and patients and caregivers were involved in this. ${ }^{29}$ The overall effect of $24 \mathrm{~h}$ telephone availability, regular home care visits, close microbiological survey and the use of MAC through tracheotomy for optimal control of respiratory secretions ${ }^{21}$ 
could explain-at least partly-the remarkably superior outcomes we had with respiratory infections. The importance of a rapid start to treatment in the prognosis of this kind of respiratory infection is well known, ${ }^{30}$ and this was something we were able to provide to our patients.

Unlike previous studies, ${ }^{8}{ }^{10-12}$ we used shared decisionmaking principles ${ }^{16}$ in the context of a deliberative model ${ }^{31}$ for the physician-patient relationship. In general, improving communication between care providers and patients can have beneficial effects on health outcomes, ${ }^{28}$ whereas defective communication may reduce optimal clinical benefit. ${ }^{28}$ Although the design of our study prevents the evaluation of the real impact of patient involvement in decision-making on survival, this could have some level of influence over the fact that our survival rate per year is higher than those found in previous studies (varying between $65 \%$ and $20 \%$ ), in which only a few of the patients included were able to make an adequately informed decision regarding their future..$^{5-12}$

Sudden death was the main cause of death in our patients with ALS with HTMV. Previous studies ${ }^{511}$ have reported sudden death as an important cause of death in ALS as the disease progresses, and autonomic disturbances with a reduction of sympathetic activities is thought to be the cause. ${ }^{32}$ At present there are no effective treatments to prevent these deaths.

No predictive factors for survival were found in our study, and each of the available previous studies found different predictors of survival after HTMV in ALS. ${ }^{8} 911$ In our opinion, survival factors can only be discussed when we have a number of studies based on excellent medical practice and following an appropriate design.

In conclusion, after being properly informed, patients with ALS who choose to maintain life with HTMV provided by a referral RCU, can have a good 1-year survival rate and respiratory problems do not have to be the cause of death. Failure to manage respiratory secretions when patients have severe $\mathrm{BD}$ must be taken into account as a cause of tracheotomy.

Competing interests JS, ES, JLD and JM have no financial relationship with any commercial entity that has an interest in the subject of this manuscript. PB is supported by a grant from the Fundación para la Investigación del Hospital Clínico sponsored by Vital Aire Inc.

Ethics approval This study was conducted with the approval of the ethics committee of the Hospital Clinico Universitario, Valencia.

Provenance and peer review Not commissioned; externally peer reviewed.

\section{REFERENCES}

1. Lechtzin N, Wiener CM, Clawson L, et al. Hospitalization in amyotrophic lateral sclerosis: causes, costs and outcomes. Neurology 2001:56:753-7.

2. Bach JR, Bianchi C, Auifero E. Oximetry and indications for tracheostomy for amyotrophic lateral sclerosis. Chest 2004;126:1502-7.

3. Bourke SC, Tomlinson M, Bullok RE, et al. Effects of non-invasive ventilation on survival and quality of life in patients with amyotrophic lateral sclerosis: a randomised controlled trial. Lancet Neurol 2006:5:140-7.

4. Sancho J, Servera E, Díaz J, et al. Efficacy of mechanical insufflation-exsufflation in medically stable patients with amyotrophic lateral sclerosis. Chest 2004;125:1400-5.

5. Hayashi H, Oppenheimer EA. ALS patients on TPPV. Totally locked-in state, neurologic findings and ethical implications. Neurology 2003;61:135-7.

6. Miller RG, Jackson CE, Kasarkis EJ, et al. Practice parameter update. The care of the patient with amyotrophic lateral sclerosis: drug, nutritional and respiratory therapies (an evidence-based review): report of the Quality Standards Subcommittee of the Academy of Neurology. Neurology 2009;73:1218-26.
7. Andersen PM, Borasio GD, Dengler R, et al. EFNS task force on management of amyotrophic lateral sclerosis: guidelines for diagnosing and clinical care of patients and relatives. An evidence-based review with good practice points. Eur J Neurol 2005; 12:921-38

8. Vianello A, Arcaro G, Palmieri A, et al. Survival and quality of life after tracheostomy for acute respiratory failure in patients with amyotrophic lateral sclerosis. J Crit Care 2011;26:329.e7-329.e14.

9. Chio A, Calvo A, Ghiglione $P$, et al. Tracheostomy in amyotrophic lateral sclerosis: a 10-year population based study in Italy. J Neurol Neurosurg Psychiatry 2010;81:1141-3.

10. Kaub-Wittemer D, von Steinbüchel N, Wasner M, et al. Quality of life and psychosocial issues in ventilated patients with amyotrophic lateral sclerosis and their caregivers. J Pain Symptom Manage 2003;26:890-6.

11. Lo Coco D, Marchese S, La Bella V, et al. The amyotrophic lateral sclerosis functional rating scale predicts survival time in amyotrophic lateral sclerosis patients on invasive mechanical ventilation. Chest 2007;132:64-9.

12. Cazzolli PA, Oppenheimer EA. Home mechanical ventilation for amyotrophic latera sclerosis: nasal compared to tracheostomy-intermittent positive pressure ventilation. J Neurol Sci 1996;139(Suppl):123-8

13. Miller RG, Rosenberg JA, Gelinas DF, et al. Practice parameter: the care of the patient with amyotrophic lateral sclerosis (an evidence-based review): report of the Quality Standards Subcommittee of the Academy of Neurology: ALS Practice Parameters Task Force. Neurology 1999:52:1311-23.

14. Bradley WG, Anderson F, Bromberg M, et al. Current managemrnt of ALS Comparison of the ALS CARE database and the AAN practice parameter. Neurology 2001:57:500-4.

15. Brooks RD, Miller RG, Swash M, et al. World Federation of Neurology Research Group on Motor Neuron Diseases. El Escorial revisited: revised criteria for the diagnosis of amyotrophic lateral sclerosis. Amyotroph Lateral Scler Other Motor Neuron Disord 2000;1:293-9.

16. Towle A, Godolphin W. Framework for teaching and learning informed shared decision making. BMJ 1999;319:766-71.

17. Sancho J, Servera E, Díaz J. Predictors of ineffective cough during a chest infection in patients with stable amyotrophic lateral sclerosis. Am J Respir Crit Care Med 2007; 175:1266-71

18. Cedarbaum JM, Stambler N, Nalta E, et al. The ALSFRS-R: a revised ALS function rating scale that incorporates assessment of respiratory function. DNF ALS Study Group (Phase III). J Neurol Sci 1999;169:13-21.

19. Norris FH, Calanchini PR, Fallat RJ, et al. The administration of guanidine in amyotrophic lateral sclerosis. Neurology 1974;24:721-8.

20. Anon. Clinical indications for noninvasive positive pressure ventilation in chronic respiratory failure due to restrictive lung disease, COPD and nocturna hypoventilation - a consensus conference report. Chest 1999;116:521-34.

21. Kohorts J, Blakely $P$, Dockter $C$, et al. AARC clinical practice guideline: long-term invasive mechanical ventilation in home -2007 revision and update. Respir Care 2007; 52:1056-62.

22. Sancho J, Servera E, Vergara $P$, et al. Mechanical insufflation-exsufflation vs. tracheal suctioning via tracheostomy tubes for patients with amyotrophic lateral sclerosis: a pilot study. Am J Phys Med Rehabil 2003;82:750-3.

23. Stupp R, Hegi ME, Mason W, et al: European Organisation for Research and Treatment of Cancer Brain Tumour and Radiation Oncology Groups; National Cance Institute of Canada Clinical Trials Group. Effects of radiotherapy with concomitant and adjuvant temozolomide versus radiotherapy alone on survival in glioblastoma in a randomised phase III study: 5 year analysis of the EORTC-NCIC trial. Lancet Neurol 2009;10:459-66

24. Trulock EP, Christie JD, Edwards LB, et al. Registry of the International Society fo Heart and Lung Transplantation: 24th official adult lung and heart lung transplant report-2007. J Heart Lung Transplant 2007:26:782-95.

25. Díaz JL, Sancho J, Servera E, et al. Ansiedad y depresión en un grupo de enfermos de ELA y sus cuidadores. Arch Bronconeumol 2009;45(Suppl):252.

26. Robbins RA, Simmons Z, Bremer BA, et al. Quality of life in ALS is maintained as physical function declines. Neurology 2001;56:442-4

27. Gauthier A, Vignola A, Calvo A, et al. Longitudinal study on quality of life and depression in ALS patient-caregiver couples. Neurology 2007:68:923-6.

28. Teutsch C. Patient-doctor communication. Med Clin North Am 2003;87:1115-45

29. Davey P, Pagliari C, Hayes A. The patient's role in the spread and control of bacterial resistance to antibiotics. Clin Microbiol Infect 2002:8(Suppl):43-68.

30. Ahmed QA, Niederman MS. Respiratory infection in the chronically critically ill patient. Ventilator associated pneumonia and tracheobronchitis. Clin Chest Med 2001;22:71-85.

31. Emanuel EJ, Emanuel LL. Four models of the physician-patient relationship. JAMA 1992:267:2221-6.

32. Asai H, Hirano M, Udaka F, et al. Sympathetic disturbances increase risk of sudden cardiac arrest in sporadic ALS. J Neurol Sci 2007:254:78-83. 